\title{
Fatores associados ao desenvolvimento de complicações precoces após artroplastia total do joelho
}

\section{Factors Associated with the Development of Early Complications after Total Knee Arthroplasty}

\author{
Matheus Rizério Tavares ${ }^{1}$ (1) Savio Diego Ribeiro de Alencar ${ }^{1}{ }^{10}$ Samir Peixoto Frazão ${ }^{1}$ \\ Matheus Lemos Azi ${ }^{1 \odot}$ David Sadgursky $^{1 \odot}$ Daniel Alencar $^{1}{ }^{\circledR}$ \\ ${ }^{1}$ Departamento de Ortopedia e Traumatologia do Hospital Manoel \\ Victorino, Salvador, BA, Brasil \\ Rev Bras Ortop 2022;57(4):661-666. \\ Endereço para correspondência Matheus Tavares, Dr, Hospital \\ Manoel Victorino, Ortopedia e Traumatologia, Praça Conselheiro \\ Almeida Couto, SN, Nazaré. Salvador, Bahia, Brasil \\ (e-mail: matheus.tavares@gmail.com).
}

\section{Resumo \\ Palavras-chave \\ - artroplastia \\ - artroplastia do joelho \\ - comorbidades \\ - complicações \\ - joelho \\ Objetivo Identificar fatores de risco para o desenvolvimento de complicações pós- operatórias locais ou clínicas ocorridas até 30 dias após a cirurgia em pacientes submetidos a artroplastia total do joelho. \\ Métodos Todos os pacientes submetidos a artroplastia de joelho realizada neste hospital no período do estudo foram incluídos na análise, baseada em dados dos prontuários dos pacientes, para investigar os fatores associados à ocorrência de complicações locais ou clínicas durante o internamento até 30 dias após o procedi- mento que necessitaram de alguma intervenção. \\ Resultados Dos 157 pacientes incluídos no estudo, 17,1\% cursaram com alguma complicação. As mais prevalentes foram artrofibrose $(6,4 \%)$, infecção profunda $(4,4 \%)$ e infecção superficial (2,5\%), dentre outras. A maior idade e maior número de comorbidades prévias foram relacionadas com maior risco de complicações pós- operatórias. \\ Conclusões A maior idade e maior número de comorbidades foram relacionados a um risco aumentado de complicações pós-operatórias. O escore Sociedade Americana de Anestesiologistas (ASA, na sigla em inglês) se mostrou insuficiente para avaliar a ocorrência de complicações pós artroplastia de joelho e novas ferramentas devem ser empregadas para este fim.}

Trabalho desenvolvido no Hospital Manoel Victorino, Salvador, BA, Brasil.

recebido

23 de Agosto de 2020

aceito

13 de Maio de 2021

Publicado on-line

Outubro 28, 2021
DOI https://doi.org/

10.1055/s-0041-1736309.

ISSN 0102-3616. (c) 2021. Sociedade Brasileira de Ortopedia e Traumatologia. All rights reserved.

This is an open access article published by Thieme under the terms of the Creative Commons Attribution-NonDerivative-NonCommercial-License, permitting copying and reproduction so long as the original work is given appropriate credit. Contents may not be used for commercial purposes, or adapted, remixed, transformed or built upon. (https://creativecommons.org/ licenses/by-nc-nd/4.0/)

Thieme Revinter Publicações Ltda., Rua do Matoso 170, Rio de Janeiro, RJ, CEP 20270-135, Brazil 

Abstract
Keywords
- arthroplasty
- arthroplasty, replacement, knee
- comorbidities
- complications
- knee

Objective To diagnose risk factors for the development of local or medical postoperative complications up to 30 days after surgery in patients undergoing total knee arthroplasty

Methods The present analysis included all patients who underwent knee arthroplasty performed in this hospital during the study period, based on data from medical records of the patients, with the aim of investigating factors related to the occurrence of local or medical complications during hospitalization and up to 30 days after the procedure, if they needed any additional intervention.

Results Out of the 157 patients included in the study, $17.1 \%$ had some complication. The most prevalent were arthrofibrosis (6.4\%), deep infection (4.4\%), and superficial infection (2.5\%), among others. Older patients and a greater number of previous comorbidities were associated with a higher risk of postoperative complications.

Conclusions Older patients and a greater number of comorbidities were related to an increased risk of postoperative complications. The American Society of Anesthesiologists (ASA) score proved to be insufficient to assess the occurrence of complications after knee arthroplasty and new tools should be used for this purpose.

\section{Introdução}

Com o aumento da expectativa de vida da população, as doenças degenerativas osteoarticulares vêm se tornando mais prevalentes e a demanda por artroplastias de substituição vem crescendo e pode chegar a 1,3 milhões de artroplastias por ano em 2030 nos Estados Unidos. ${ }^{1}$ Os avanços das técnicas cirúrgicas, o desenvolvimento de novos materiais, as melhoras incrementais na seleção de pacientes e nos regimes de reabilitação permitiram que as artroplastias de joelho apresentem melhores resultados funcionais e melhor qualidade de vida após a cirurgia, assim como internações mais curtas. ${ }^{1-3}$

As complicações após artroplastias totais de joelho (ATJs) estão associadas a uma maior morbidade, além de um aumento na permanência hospitalar, nos custos de tratamento e piores resultados funcionais. Foram consideradas complicações precoces os eventos ocorridos até 30 dias após o procedimento inicial que necessitam de reinternação, prolongamento da internação ou terapias especificas. As complicações nas cirurgias de artroplastia são diversas e muitas delas, de menor gravidade e que não retardaram a alta ou que não necessitaram de intervenção especifica, não foram consideradas. ${ }^{4}$ Dentre as complicações pós-operatórias, as infecções podem ser devastadoras, especialmente as infecções profundas, gerando internações prolongadas e novos procedimentos ou cirurgias de revisão. ${ }^{5}$ Além destes, podem ocorrer solturas assépticas, artrofibrose, tromboembolismo venoso e eventos cardiovasculares desencadeados pelo trauma cirúrgico, além dos relacionados à internação hospitalar. $^{6}$

Frequentemente, são utilizados parâmetros e escores para identificar os pacientes de maior risco para ocorrência de complicações após o tratamento cirúrgico, como o escore da Sociedade Americana de Anestesiologistas (ASA, na sigla em inglês). Comorbidades clínicas, estimativas de tempo cirúrgico, idade e índice de massa corporal IMC também são avaliados. ${ }^{4,7-9}$ No entanto, novos estudos podem melhorar a capacidade do cirurgião de identificar pacientes com maior risco de complicações pós-operatórias para que elas sejam manejadas adequadamente com o uso de diferentes técnicas e estratégias. $^{10}$

O objetivo primário do presente estudo foi identificar fatores de risco para o desenvolvimento de complicações pós-operatórias precoces (até 30 dias após a cirurgia) em pacientes submetidos a ATJ.

\section{Método}

No presente estudo observacional do tipo coorte retrospectiva, foram analisados os registros médicos de pacientes submetidos a ATJ em nosso serviço. O presente estudo foi aprovado pelo comitê de ética em pesquisa. Foram incluídos pacientes submetidos a ATJ entre 1 de outubro de 2016 e 31 de agosto de 2018 com dados de prontuário completos. Pacientes operados inicialmente em outros serviços que necessitaram de cirurgia de revisão devido a complicações foram excluídos do estudo. Foram analisados dados demográficos como gênero e idade, fatores relacionados ao tratamento como tempo de internamento, lateralidade e tipo de implante, comorbidades clinicas prévias, escore ASA précirúrgico, ${ }^{11}$ classificação radiográfica da gonartrose, ${ }^{12}$ deformidade inicial, uso de dreno suctor e ocorrência de complicações clínicas como infecções do trato urinário, tromboembolismo venoso, injúria renal aguda e complicações cardiovasculares, além de complicações cirúrgicas locais como artrofibrose, infecções superficiais e profundas que necessitaram de desbridamento ou cirurgia de revisão, além de dor refratária nos primeiros 30 dias após a cirurgia. ${ }^{4}$ Foram considerados como dor refratária os casos nos quais o quadro álgico referido pelo paciente retardou a alta hospitalar ou comprometeu a reabilitação fisioterápica nos primeiros 30 dias após a cirurgia. Foram considerados casos de artrofibrose os pacientes que cursaram com rigidez com 
amplitude de movimento de flexão ativa $<90^{\circ}$ e $>10^{\circ}$ de contratura em flexão que necessitaram de internamento para a realização de manipulação articular sob anestesia. ${ }^{13}$ Complicações que ocorreram > 30 dias após a cirurgia foram consideradas tardias e foram excluídas do presente estudo. Complicações de menor gravidade que não necessitaram de intervenção e não retardaram a alta do paciente não foram incluídas no estudo.

A gravidade da gonartrose foi classificada conforme a classificação radiográfica de Ahlback para osteoartrite do joelho.

\section{Resultados}

Um total de 157 pacientes foram selecionados para o estudo. Destes, 35 (22,3\%) eram do sexo masculino. A idade dos pacientes variou entre 35 e 84 anos (média 66,3; $\sigma=8,03$ ). A maioria dos pacientes era do grupo ASA II, e apenas 33 (21\%) dos pacientes não apresentavam comorbidades prévias. A comorbidade mais prevalente foi hipertensão arterial sistêmica, seguida de diabetes mellitus e artrite reumatoide, conforme observado na - Tabela 1. Todos os joelhos apresentaram algum grau de deformidade do eixo mecânico, sendo a deformidade em varo a mais frequente $(89,2 \%$; $n=140)$ e joelhos valgos totalizaram $10,8 \%(n=17)$. A gravidade da osteoartrite foi classificada segundo a classificação de Ahlback e o grau III incluiu 3,2\% $(n=5)$, grau IV 59,9\% $(n=54)$ e grau V 36,9\% $(n=58)$ dos pacientes. Os casos de bilateralidade de gonartrose totalizaram 88 pacientes (56,1\%). Nos casos de gonartrose unilateral, não houve diferença significativa de lateralidade, conforme observado na - Tabela 2. Os pacientes ficaram internados de 2 a 98 dias (média 5,$38 ; \sigma=7,62$ ) e os pacientes com mais comorbidades demandaram internações mais prolongadas $(p=0,026)$.

Das 157 ATJs realizadas no período do estudo, 17,1\% ( $n=27$ ) apresentaram alguma complicação pós-operatória (-Tabela 3). A complicação mais frequente foi a artrofibrose ( $n=10,6,4 \%)$, seguida de infecção profunda $(n=7 ; 4,4 \%)$

Os pacientes apresentaram em média 1,18 comorbidades $(\sigma=0,84)$, e o número de comorbidades foi relacionado à ocorrência de complicações. O grupo que apresentou complicações pós-operatórias tinha mais comorbidades prévias

Tabela 1 Estatísticas descritivas e perfil dos pacientes

\begin{tabular}{|c|c|c|c|c|c|c|c|c|}
\hline \multirow{2}{*}{$\begin{array}{l}\text { Variáveis } \\
\text { Gênero }\end{array}$} & \multirow[b]{2}{*}{ Feminino } & \multirow{2}{*}{$\begin{array}{l}n \\
122\end{array}$} & \multirow{2}{*}{$\begin{array}{l}\% \\
77,7\end{array}$} & \multicolumn{2}{|c|}{ Complicação (\%) } & \multicolumn{2}{|c|}{$\begin{array}{l}\text { Sem Complica- } \\
\text { ção (\%) }\end{array}$} & \multirow{2}{*}{$\begin{array}{l}\text { Significância }(p) \\
0,992^{a}\end{array}$} \\
\hline & & & & 101 & $(82,8 \%)$ & 21 & $(17,2 \%)$ & \\
\hline & Masculino & 35 & 22,3 & 29 & $(82,9 \%)$ & 6 & $(17,1 \%)$ & \\
\hline \multirow[t]{2}{*}{ Idade } & $<70$ anos & 65 & 41,4 & 0 & $(0,0 \%)$ & 65 & $(100,0 \%)$ & $<0,001^{c}$ \\
\hline & $\geq 70$ anos & 92 & 58,5 & 27 & $(29,3 \%)$ & 65 & $(70,7 \%)$ & \\
\hline \multirow[t]{4}{*}{ Escore ASA } & I & 32 & 20,4 & 28 & $(87,5 \%)$ & 4 & $(12,5 \%)$ & $0,881^{a}$ \\
\hline & II & 105 & 66,9 & 86 & $(81,9 \%)$ & 19 & $(18,1 \%)$ & \\
\hline & III & 19 & 12,1 & 15 & $(78,9 \%)$ & 4 & $(21,1 \%)$ & \\
\hline & IV & 1 & 0,6 & 1 & $(100,0 \%)$ & 0 & $(0,0 \%)$ & \\
\hline \multirow[t]{5}{*}{ Número de comorbidades } & Nenhuma & 33 & 21 & 29 & $(87,9 \%)$ & 4 & $(12,1 \%)$ & $0,045^{b}$ \\
\hline & 1 & 73 & 46,5 & 62 & $(84,9 \%)$ & 11 & $(15,1 \%)$ & \\
\hline & 2 & 42 & 26,8 & 34 & $(81,0 \%)$ & 8 & $(19,0 \%)$ & \\
\hline & 3 & 8 & 5,1 & 5 & $(62,5 \%)$ & 3 & $(37,5 \%)$ & \\
\hline & 4 & 1 & 0,6 & 0 & $(0,0 \%)$ & 1 & $(100,0 \%)$ & \\
\hline \multicolumn{2}{|l|}{ Hipertensão arterial } & 111 & 71,3 & 91 & $(82,0 \%)$ & 21 & $(18,9 \%)$ & $0,416^{a}$ \\
\hline \multicolumn{2}{|l|}{ Diabetes mellitus } & 36 & 22,9 & 26 & $(72,2 \%)$ & 10 & $(27,8 \%)$ & $0,055^{\mathrm{a}}$ \\
\hline Cardiopatia & & 6 & 3,8 & 4 & $(66,7 \%)$ & 2 & $(33,3 \%)$ & $0,286^{a}$ \\
\hline \multicolumn{2}{|l|}{ Artrite reumatoide } & 8 & 5,1 & 6 & $(75,0 \%)$ & 2 & $(25,0 \%)$ & $0,414^{a}$ \\
\hline Obesos & & 4 & 2,5 & 4 & $(100,0 \%)$ & 0 & $(0,0 \%)$ & $0,466^{a}$ \\
\hline \multicolumn{2}{|l|}{ Hipotireoidismo } & 4 & 2,5 & 3 & $(75,0 \%)$ & 1 & $(25,0 \%)$ & $0,675^{a}$ \\
\hline \multicolumn{2}{|l|}{ Transtorno psiquiátrico } & 4 & 2,5 & 4 & $(100,0 \%)$ & 0 & $(0,0 \%)$ & $0,356^{\mathrm{a}}$ \\
\hline \multicolumn{2}{|l|}{ Insuficiência renal } & 2 & 1,3 & 1 & $(50,0 \%)$ & 1 & $(50,0 \%)$ & $0,216^{a}$ \\
\hline Asma & & 2 & 1,3 & 1 & $(50,0 \%)$ & 1 & $(50,0 \%)$ & $0,216^{a}$ \\
\hline Hepatite C & & 1 & 0,6 & 1 & $(100,0 \%)$ & 0 & $(0,0 \%)$ & $0,648^{a}$ \\
\hline
\end{tabular}

Abreviação: ASA, Sociedade Americana de Anestesiologistas

${ }^{a}$ Teste exato de Fisher

${ }^{\mathrm{b}}$ Teste tau-b de Kendall

'Teste $t$ de Student 
664 Fatores associados a complicações após artroplastia total do joelho Tavares et al.

Tabela 2 Estatísticas descritivas das comorbidades observadas e tratamento realizado

\begin{tabular}{|l|l|l|l|l|l|l|l|l|}
\hline Variáveis & & $\boldsymbol{n}$ & \multicolumn{3}{l|}{ Complicação (\%) } & \multicolumn{2}{l|}{$\begin{array}{l}\text { Sem Complicação } \\
(\%)\end{array}$} & Significância $(p)$ \\
\hline Gonartrose & Bilateral & 88 & 56,1 & 75 & $(85,20 \%)$ & 13 & $(14,80 \%)$ & 0,366 \\
\hline & Unilateral & 69 & 43,9 & 55 & $(79,70 \%)$ & 14 & $(20,30 \%)$ & \\
\hline Classificação de Ahlback & III & 5 & 3,2 & 3 & $(60,00 \%)$ & 2 & $(40,00 \%)$ & 0,258 \\
\hline & IV & 94 & 59,9 & 83 & $(88,30 \%)$ & 11 & $(11,70 \%)$ & \\
\hline & V & 58 & 36,9 & 44 & $(75,90 \%)$ & 14 & $(24,10 \%)$ & \\
\hline Deformidade & Varo & 140 & 89,2 & 116 & $(82,90 \%)$ & 24 & $(17,10 \%)$ & 0,959 \\
\hline & Valgo & 17 & 10,8 & 14 & $(82,40 \%)$ & 3 & $(17,60 \%)$ & \\
\hline Prótese & Primária & 149 & 94,9 & 123 & $(82,60 \%)$ & 26 & $(17,40 \%)$ & 0,72 \\
\hline & Revisão & 8 & 5,1 & 7 & $(87,50 \%)$ & 1 & $(12,50 \%)$ & \\
\hline Lateralidade da cirurgia & Esquerdo & 86 & 54,8 & 71 & $(82,60 \%)$ & 15 & $(17,40 \%)$ & 0,929 \\
\hline & Direito & 71 & 45,2 & 59 & $(83,10 \%)$ & 12 & $(16,90 \%)$ & \\
\hline Dreno suctor & Utilizado & 140 & 89,2 & 27 & $(19,30 \%)$ & 113 & $(80,70 \%)$ & 0,047 \\
\hline & Não Utilizado & 17 & 10,8 & 0 & $(0,00 \%)$ & 17 & $(100,00 \%)$ & \\
\hline
\end{tabular}

Tabela 3 Frequências das complicações pós-operatórias agudas

\begin{tabular}{|l|l|l|}
\hline Complicações & Frequência & Porcentagem \\
\hline Sem complicações & 130 & 82,8 \\
\hline Artrofibrose & 10 & 6,4 \\
\hline Infecção profunda & 7 & 4,4 \\
\hline Infecção superficial & 4 & 2,5 \\
\hline Dor persistente & 3 & 0,6 \\
\hline Fratura periprotética & 1 & 0,6 \\
\hline Injuria renal aguda & 1 & 0,6 \\
\hline Infecção urinária & 1 & 0,6 \\
\hline Total & 157 & 100,0 \\
\hline
\end{tabular}

(média de 1,5) em relação ao grupo sem complicações (média de 1,1$)(p=0,04)$. Os pacientes mais idosos apresentaram mais comorbidades $(p<0,001)$ e cursaram com mais complicações ( $p=0,045)$. Os pacientes com mais comorbidades necessitaram de internamentos mais prolongados e apresentaram mais complicações, como pode-se observar na - Figura 1. Apesar dos pacientes diabéticos apresentarem mais complicações pós-operatórias (os ratio $[\mathrm{OR}]=2,35$ ) a correlação não chegou a ser significante $(p=0,055)$.

Na maioria das cirurgias $(89,2 \%, n=140)$ foi utilizado dreno custo. Do grupo em que o dreno não foi utilizado (10,8\%; $n=17)$, nenhum paciente apresentou complicações pós-operatórias, e esta diferença foi estatisticamente significativa ( $p=0,047 ; \mathrm{OR}=8,48)$.

O tipo de implante, escore ASA, deformidade, classificação da gonartrose, tempo de internamento e gênero não foram estatisticamente significativas para a ocorrência de complicações.

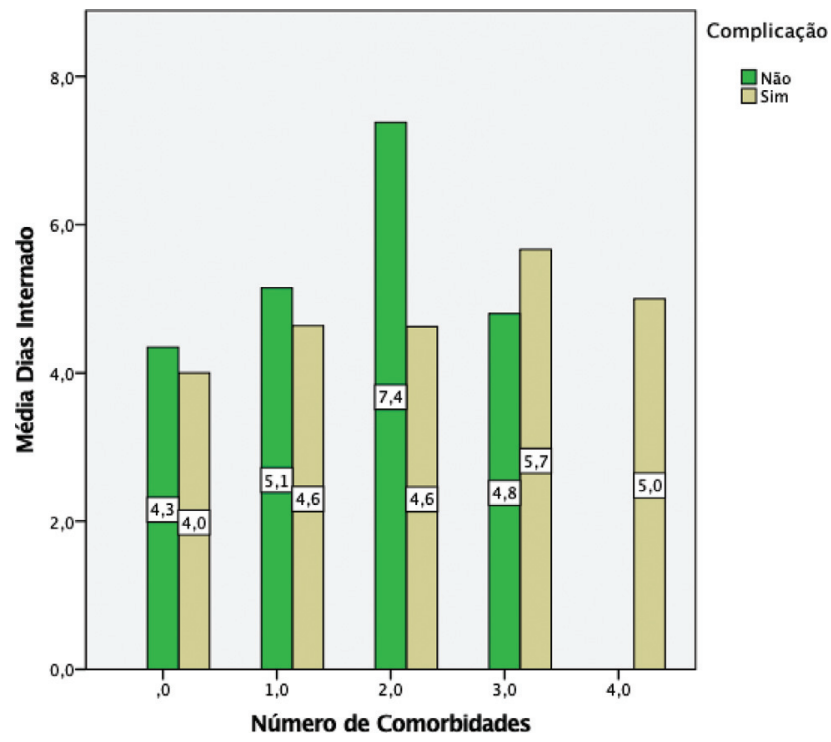

Fig. 1 Comparação entre número de comorbidades e média de dias internado por grupo de complicações.

\section{Discussão}

Diversos autores estratificam os pacientes pelo escore ASA. Apesar dos pacientes classificados como ASA 3 ou superior apresentarem um maior risco de complicações, grande parte dos pacientes são classificados como ASA 2, diminuindo a relevância do escore, pois ele não contempla o número de comorbidades. Foi observado que o número de comorbidades aumentou o risco de complicações e este dado deve ser considerado na avaliação pré-operatória do paciente ${ }^{1,6-8,14}$ Foi também observado que pacientes com maiores idades tendem a ter mais complicações, e estudos recentes evidenciam que com > 80 anos são de risco para a ocorrência de complicações. No entanto, a literatura sugere que a presença 
de comorbidades parece ser mais relevante do que a idade isoladamente. ${ }^{10}$ Dentre as comorbidades, vale notar que os pacientes portadores de diabetes mellitus apresentaram mais complicações em geral segundo dados da literatura, especialmente as complicações relacionadas com deiscência de feridas e infecções. ${ }^{15,16}$ Observou-se no presente estudo que pacientes cardiopatas apresentaram maior risco de desenvolver artrofibrose, provavelmente por dificuldade de realizar uma reabilitação adequada, mas novos estudos devem ser realizados para investigas tal associação. ${ }^{3,13}$ Nenhuma outra comorbidade foi capaz de alterar o risco de complicações em geral com significância estatística independentemente, provavelmente devido ao pequeno tamanho amostral dos subgrupos destas patologias na amostra observada.

Nenhum dos pacientes do grupo em que não foi colocado dreno suctor apresentou complicação, o que foi estatisticamente significativo. Não é possível afirmar com o presente estudo se o dreno é um fator de risco ou se este recurso não foi utilizado quando o cirurgião se sentiu confortável em não colocar o dispositivo devido à boa hemostasia obtida e tempo operatório curto em pacientes sem comorbidades significativas, e estes achados devem ser considerados com cautela. A literatura sugere que o tempo operatório prolongado aumenta a chance de infecção e cirurgias de maior morbidade aumentam a dor pós-operatória e dificultam a reabilitação. ${ }^{3}$

A complicação mais frequente foi a artrofibrose, de acordo com observações de outros autores. Sua incidência pode estar relacionada a uma reabilitação de má qualidade e analgesia insatisfatória no pós-operatório, visto que estes fatores são classicamente relacionados à gênese desta patologia. $\mathrm{O}$ tratamento da artrofibrose demanda reabilitação fisioterápica agressiva e analgesia multimodal nos estágios iniciais, sendo necessária reinternação para a realização de manipulação articular ou até mesmo cirurgias de revisão em casos refratários. Novos estudos são necessários para elucidar se os pacientes atendidos pelo sistema único de saúde têm dificuldade de acesso a reabilitação fisioterápica adequada e acesso a analgesia multimodal. ${ }^{3,13}$

Não se observou correlação significativa entre ocorrência de complicações agudas e obesidade, mas dados da literatura apontam que pacientes obesos, especialmente os que têm IMC $\geq 40$ estão mais susceptíveis a complicações como deiscência de feridas e dificuldade na reabilitação. ${ }^{2}$ A classificação radiográfica, deformidade e emprego de próteses de revisão não foram significativos para a ocorrência de complicações na amostra estudada, mas a literatura sugere que o tempo cirúrgico prolongado, esperado em casos de artrose mais grave com falhas ósseas ou cirurgias de revisão, influencie negativamente os resultados, elevando principalmente o risco de infecção. ${ }^{14}$

A duração da internação foi em média 5,3 dias. No entanto, há uma tendência global a buscar tempos de internação menores para a redução de custo de internação e complicações relacionados à colonização por bactérias hospitalares, e estudos recentes evidenciam esta queda progressiva do tempo de internação na literatura internacional. ${ }^{1}$ Ainda não há consenso se a realização do procedimento de forma ambulatorial reduza a ocorrência de complicações. ${ }^{17-19}$ Protocolos bem definidos de reabilitação e analgesia, além de equipe multidisciplinar especializada parecem ser cruciais para o sucesso do procedimento de forma ambulatorial.

O presente estudo apresenta limitações, especialmente por ser unicêntrico e retrospectivo baseado em dados de prontuário. $O$ pequeno número de indivíduos em subgrupos específicos dificulta a análise estatística. Outra possível limitação que deve ser considerada é que, por se tratar de um centro de referência ortopédico de alta complexidade, casos de deformidades mais graves e pacientes com comorbidades clínicas significativas sejam referenciados para o mesmo, aumentando indiretamente o risco de complicações e tempo de internação.

\section{Conclusão}

A idade e o número de comorbidades aumentaram o risco de complicações pós-operatórias na população estudada. Novos estudos são necessários para elucidar se as comorbidades influenciam mais nos resultados do que a idade dos pacientes isoladamente, visto que os pacientes mais velhos tendem a ter um maior número de comorbidades e dificultam a análise dos resultados. O escore ASA, amplamente utilizado para avaliar o risco cirúrgico dos pacientes, é insuficiente para estratificar o risco de complicações dos pacientes, visto que não contempla o número de comorbidades clínicas ou a idade em sua avaliação. Escores mais abrangentes como o SF-36 ou outras ferramentas podem se mostrar mais confiáveis para estimar o risco de complicações após ATJs, mas novos estudos são necessários para elucidar este questionamento.

\section{Suporte Financeiro}

Não houve suporte financeiro de fontes públicas, comerciais, ou sem fins lucrativos.

Conflito de Interesses

Os autores declaram não haver conflito de interesses.

\section{Referências}

1 Sarpong NO, Boddapati V, Herndon CL, Shah RP, Cooper HJ, Geller JA. Trends in Length of Stay and 30-Day Complications After Total Knee Arthroplasty: An Analysis From 2006 to 2016. J Arthroplasty 2019;34(08):1575-1580

2 Wagner ER, Kamath AF, Fruth K, Harmsen WS, Berry DJ. Effect of Body Mass Index on Reoperation and Complications After Total Knee Arthroplasty. J Bone Joint Surg Am 2016;98(24): 2052-2060

3 Cheuy VA, Foran JRH, Paxton RJ, Bade MJ, Zeni JA, Stevens-Lapsley JE. Arthrofibrosis Associated With Total Knee Arthroplasty. J Arthroplasty 2017;32(08):2604-2611

4 Frosch P, Decking J, Theis C, Drees P, Schoellner C, Eckardt A. Complications after total knee arthroplasty: a comprehensive report. Acta Orthop Belg 2004;70(06):565-569

5 Pecora JR, Lima ALM, Helito CP, Gobbi RG, Demange MK, Camanho GL. Protocol for treating acute infections in cases of total knee arthroplasty. Acta Ortop Bras 2019;27(01):27-30 
6 Lehtonen EJ, Hess MC, McGwin G Jr, Shah A, Godoy-Santos AL, Naranje S. Risk factors for early hospital readmission following total knee arthroplasty. Acta Ortop Bras 2018;26(05):309-313

7 Basques BA, Bell JA, Fillingham YA, Khan JM, Della Valle CJ. Gender Differences for Hip and Knee Arthroplasty: Complications and Healthcare Utilization. J Arthroplasty 2019;34(08):1593-1597.e1

8 Feng B, Lin J, Jin J, Qian WW, Wang W, Weng XS. Thirty-day Postoperative Complications following Primary Total Knee Arthroplasty: A Retrospective Study of Incidence and Risk Factors at a Single Center in China. Chin Med J (Engl) 2017;130(21):2551-2556

9 George J, Piuzzi NS, Ng M, Sodhi N, Khlopas AA, Mont MA. Association Between Body Mass Index and Thirty-Day Complications After Total Knee Arthroplasty. J Arthroplasty 2018;33(03): 865-871

10 Souza GGA, Ramalho RSC, Albuquerque RSPE, Barretto JM, Chaves RSM, de Sousa EB. Higher risk of complications after total knee arthroplasty in octogenarians. Acta Ortop Bras 2020;28(04):177-181

11 Saklad MMD. Grading of Patients for Surgical Procedures. Anesthesiol J Am Soc Anesthesiol 1941;2(03):281-284

12 Petersson IF, Boegård T, Saxne T, Silman AJ, Svensson B. Radiographic osteoarthritis of the knee classified by the Ahlbäck and Kellgren \& Lawrence systems for the tibiofemoral joint in people aged 35-54 years with chronic knee pain. Ann Rheum Dis 1997;56 (08):493-496
13 Thompson R, Novikov D, Cizmic Z, et al. Arthrofibrosis After Total Knee Arthroplasty: Pathophysiology, Diagnosis, and Management. Orthop Clin North Am 2019;50(03):269-279

14 George J, Mahmood B, Sultan AA, et al. How Fast Should a Total Knee Arthroplasty Be Performed? An Analysis of 140,199 Surgeries. J Arthroplasty 2018;33(08):2616-2622

15 Pugely AJ, Callaghan JJ, Martin CT, Cram P, Gao Y. Incidence of and risk factors for 30-day readmission following elective primary total joint arthroplasty: analysis from the ACS-NSQIP. J Arthroplasty 2013;28(09):1499-1504

16 Helito CP, Sobrado MF, Giglio PN, et al. The use of negativepressure wound therapy after total knee arthroplasty is effective for reducing complications and the need for reintervention. BMC Musculoskelet Disord 2020;21(01):490

17 Arshi A, Leong NL, D'Oro A, et al. Outpatient Total Knee Arthroplasty Is Associated with Higher Risk of Perioperative Complications. J Bone Joint Surg Am 2017;99(23):1978-1986

18 Bordoni V, Poggi A, Zaffagnini S, Previtali D, Filardo G, Candrian C. Outpatient total knee arthroplasty leads to a higher number of complications: a meta-analysis. J Orthop Surg Res 2020;15(01): 408

19 Crawford DA, Adams JB, Berend KR, Lombardi AV Jr. Low complication rates in outpatient total knee arthroplasty. Knee Surg Sports Traumatol Arthrosc 2020;28(05):1458-1464 